\title{
Use of Adjuvant Chemotherapy in Patients with Advanced Bladder Cancer after Neoadjuvant Chemotherapy
}

\author{
Wilson Sui ${ }^{\mathrm{a}}$, Emerson A. Lim ${ }^{\mathrm{b}}$, G. Joel Decastro ${ }^{\mathrm{a}}$, James M. McKiernan ${ }^{\mathrm{a}}$ \\ and Christopher B. Anderson ${ }^{\mathrm{a}, *}$ \\ ${ }^{a}$ Department of Urology, Columbia University Medical Center, New York, NY, USA \\ ${ }^{\mathrm{b}}$ Department of Medicine, Columbia University Medical Center, New York, NY, USA
}

\begin{abstract}
.
Objectives: To compare the outcomes of adjuvant chemotherapy (AC) versus observation in patients with non-organ confined disease after neoadjuvant chemotherapy and radical cystectomy (RC).

Materials and methods: Using the National Cancer Database, we identified patients who received NAC prior to RC and had advanced stage (pT3/4) or pathologically involved nodes (pN+) at the time of surgery from 2004-2013. We determined whether patients then received AC or were managed with observation only and used multivariable proportional hazards regression to estimate the impact of $\mathrm{AC}$ on overall survival.

Results: Overall $34 \%(\mathrm{~N}=705)$ of patients who received NAC and underwent RC were pT3/4 and/or pN+. Of these patients, $24 \%(\mathrm{~N}=168)$ received subsequent chemotherapy and the rest were observed. Median survival for the entire cohort was 21 months (IQR 12-45). There was not a statistically significant difference in median survival between the AC and observation groups (23 months [IQR 14-46] versus 20 months [IQR 12-46], log-rank $p=0.52$ ). On multivariate analysis there was no survival advantage for the AC cohort. Subgroup analysis of $\mathrm{pN}+$ patients who received $\mathrm{AC}$ also did not show a survival advantage.

Conclusions: Patients who are pT3/4 and/or $\mathrm{pN}+$ after NAC and $\mathrm{RC}$ have a poor prognosis. The addition of AC does not seem to be beneficial. Further research should focus identifying patients who may benefit from additional chemotherapy.
\end{abstract}

Keywords: Urinary bladder neoplasms, chemotherapy, adjuvant, neoadjuvant therapies, lymph nodes

\section{ABBREVIATIONS}

$\mathrm{AC}$

CCI

$\mathrm{LN}$ adjuvant chemotherapy

Charlson comorbidity index

$$
\text { lymph node }
$$

$\begin{array}{ll}\text { NCDB } & \text { National Cancer Database } \\ \text { NAC } & \text { Neoadjuvant chemotherapy } \\ \text { RC } & \text { Radical cystectomy } \\ \text { UC } & \text { Urothelial carcinoma }\end{array}$

\section{INTRODUCTION}

Platinum-based neoadjuvant chemotherapy (NAC) is associated with improved survival among patients with invasive bladder cancer having radical
Correspondence to: Christopher B. Anderson, Department Urolumbia University Medical Center, Herbert Irving Pavilion, 161 Fort Washington, 11th Floor, New York, NY 10032 USA. Tel.: +1 212305 5311; Fax: +1 212342 6870; E-mail: cba2125@cumc.columbia.edu. 
cystectomy (RC) [1-3] and is recommended by clinical guidelines [4]. Neoadjuvant chemotherapy has the advantage of treating micrometastatic disease prior to surgery when systemic chemotherapy is likely best tolerated [5, 6]. Despite the significant improvement of primary tumor downstaging with NAC, up to half of patients are ultimately found to have locally advanced disease and 20\% have lymph node (LN) metastases at RC $[1,7,8]$. These patients have a poor prognosis and most die of cancer [9].

Cisplatin-based adjuvant chemotherapy (AC) is an option for patients with advanced disease at RC. On meta-analysis of 945 patients from 9 randomized trials, the majority of whom had $\geq \mathrm{pT} 3$ disease and about a third of whom were $\mathrm{pN}+, \mathrm{AC}$ was associated with a $23 \%$ decreased risk of death. The effect of AC on disease free-survival was especially apparent in trials with higher nodal involvement [10]. Results from a more recent large randomized AC trial failed to observe a survival benefit among all patients, but did suggest that those who were pN0 may benefit from immediate AC [11].

However, the indication for and potential benefit of $\mathrm{AC}$ is unclear in patients who have previously been treated with systemic NAC, as these patients may have chemotherapy resistant disease. Two small retrospective studies provide contrasting reports about whether AC benefits patients who have advanced disease after $\mathrm{NAC}$ and $\mathrm{RC}[7,12]$. Yet some physicians recommend $\mathrm{AC}$ as demonstrated by a recent survey of urologic oncologists from the UK, which found that $55 \%$ of respondents would give AC after NAC to this patient population [13]. For those physicians who would give $\mathrm{AC}$, most would administer additional multi-agent cisplatin-based chemotherapy. Current guidelines do not recommend $\mathrm{AC}$ in patients with advanced disease after NAC [14].

With the increasing utilization of NAC, there is a need to better understand the prognosis and treatment options for those with advanced cancer despite having received systemic chemotherapy [15]. We hypothesized that among patients with locally advanced or lymph node positive disease at RC after NAC, multiagent $\mathrm{AC}$ was associated with improved survival. We further hypothesized that in patients with lymph node positive $(\mathrm{pN}+)$ disease, those with lower tumor burden ( 2 or less positive lymph nodes) would respond better to AC than those with higher tumor burden (more than 2 positive lymph nodes).

\section{MATERIALS AND METHODS}

\section{Dataset}

Beginning in 2004, the NCDB has captured over $70 \%$ of new annual cancer diagnoses. This data is collected from over 1,500 programs participating in the American College of Surgeons Commission on Cancer approvals program. The NCDB captures information on patient demographic characteristics, facility descriptors, cancer-specific information, treatment modality and mortality.

\section{Cohort}

We identified all patients diagnosed with bladder cancer between 2004 and 2013 using the International Classification of Diseases-Oncology, 3rd edition (ICO-3) topography codes C67.1-67.9 (Fig. 1). Urothelial carcinoma (UC) histology was defined by ICO-3 codes 8120 and 8130 [16]. We identified all patients who were treated with $\mathrm{RC}(\mathrm{N}=47,207)$ and then excluded patients with another primary cancer diagnosis $(\mathrm{N}=20,315)$.

Neoadjuvant chemotherapy was defined as receipt of chemotherapy less than 6 months prior to RC and utilization of subsequent $\mathrm{AC}$ was identified using the temporal relation variable as detailed by the NCDB participant use file [17]. We only considered multiagent chemotherapy and excluded patients who were treated with single agent perioperative chemotherapy or chemotherapy with an unknown number of agents. Among patients who were treated with NAC prior to $\mathrm{RC}(\mathrm{N}=3,003)$, we excluded patients who were clinically node positive $(\mathrm{N}=365)$, patients who were $\mathrm{cM}+/ \mathrm{MX}$ at diagnosis $(\mathrm{N}=125)$ and use of single agent or unknown number of chemotherapeutic agents $(\mathrm{N}=370)$. We then identified patients with pathologically involved lymph nodes $(\mathrm{pN}+)$ and/or locally advanced disease (pT3 or pT4) on final surgical pathology after RC $(n=723)$. Finally we eliminated all patients who were deceased within 6 months of surgery $(n=18)$ for a final analytic cohort of $\mathrm{N}=705$ patients.

\section{Outcome}

Our primary outcome was overall survival after diagnosis of bladder cancer. 


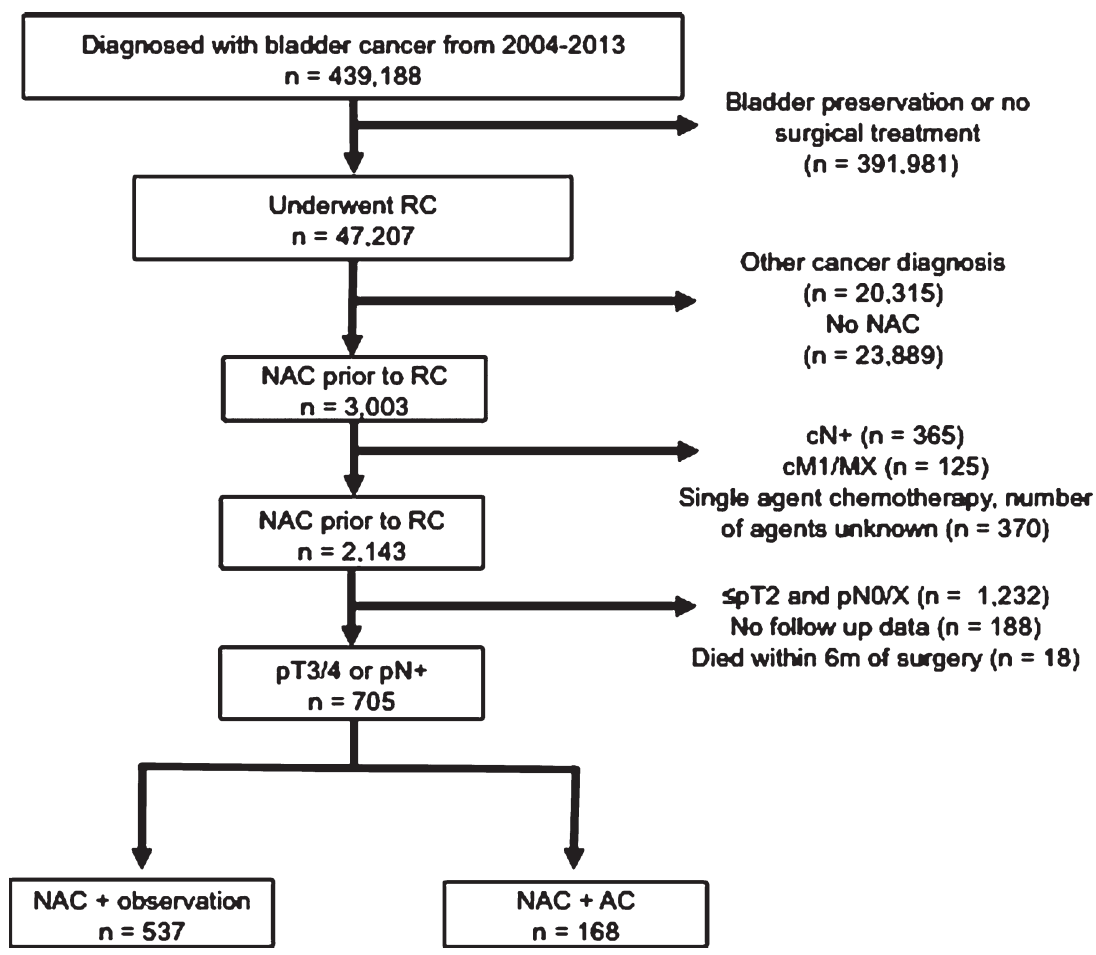

Fig. 1. Inclusion and exclusion criteria of pT3/4 or pN+ patients who underwent NAC and RC from the 2004-2013 NCDB.

\section{Independent variables}

The independent variables were analyzed as designated by the Participant Use File dictionary [17]. These included sex, age, race (Caucasian, African American, other), insurance status, modified Charlson-Deyo score (CCI), residence location, county-level information and hospital characteristics. The CCI was calculated based on up to ten ICD-9CM secondary diagnosis codes. County-level income data was abstracted from 2000 and 2012 U.S. Census. Hospitals were described by their type based on Commission on Cancer Accreditation program classification system and geographic location. The characteristics of the lymph node dissection included the number of lymph nodes examined and the number that was pathologically involved.

\section{Analysis}

Demographic and disease characteristics were compared by treatment type using chi-square tests for categorical variables and Student's $t$-tests for continuous variables. We generated survival curves using the Kaplan-Meier method and compared survival between treatment groups using the log-rank test. We used proportional hazards regression to test our hypothesis that $\mathrm{AC}$ is associated with improved survival. Our model included age, sex, race, CCI, pathologic T stage, number of lymph nodes removed, number of pathologically involved lymph nodes and tumor histology to adjust for confounding. The proportional hazards assumption was tested graphically for each covariate. We performed a sensitivity analysis to determine if the effect of AC was similar after excluding patients with non-urothelial histology $(\mathrm{N}=94)$.

We performed a subgroup analysis of only patients who were $\mathrm{pN}+$. We categorized patients according to the number of positive lymph nodes ( $\leq 2$ versus $>2$ ) and plotted Kaplan-Meier curves for the two treatment groups. The cutpoint of 2 lymph nodes was chosen as this was the median number of positive lymph nodes in previous reports $[18,19]$. We built a multivariable model that included an interaction term (number of positive lymph nodes $x$ treatment type) to allow for a non-linear association between these two variables. All analyses were performed using SPSS v23.0. $P$-values $<0.05$ were considered statistically significant. 


\section{RESULTS}

Of the 2,143 patients who received multiagent NAC prior to $\mathrm{RC}, 33 \%(\mathrm{~N}=705)$ patients had $\mathrm{pT} 3 / 4$ or $\mathrm{pN}+$ at the time of cystectomy. Of these patients, $24 \%(\mathrm{~N}=168)$ received AC while $76 \%(\mathrm{~N}=537)$ were observed with no additional chemotherapy. Demographic and tumor specific characteristics are shown in Table 1. The AC group had a lower proportion treated at an academic hospital. The distribution of tumor stage was similar across both groups. There was no difference in the number of lymph nodes examined or number of pathologically involved lymph nodes between groups, although the AC group was more likely to be $\mathrm{pN}+$.

Follow-up for the entire cohort was 44 months (IQR 29-66) and median overall survival was 21 months (IQR 12-45). Compared to the observation group, the AC group had longer median survival (Fig. 2A), however this was not statistically significant (23 months [IQR 14-46] versus 20 months [IQR 12-46], log-rank $p=0.52$ ). On multivariable analysis (Table 2), receipt of AC was not associated with decreased hazards of death (HR $0.91,95 \%$ CI $0.72-1.14 ; p=0.42$ ). When patients with non-UC histology were excluded $(\mathrm{N}=94)$, receipt of AC was still not associated with improved survival (HR $0.87,95 \%$ CI $0.65-1.12 ; p=0.36$ ).

On subgroup analysis of $\mathrm{pN}+$ patients $(n=337)$, median survival for the AC group was 22 months (IQR 14-33) and the observation group 17 months (IQR 11-32, log-rank $p=0.23$; Fig. 2B). We grouped patients according to more or less than 2 positive lymph nodes (Fig. 3). Neither the $\leq 2 \mathrm{LN}$ group (logrank $p=0.20$ ) nor the $>2$ positive $L N$ group (log-rank $p=0.92$ ) demonstrated a statistically significant survival advantage with receipt of AC. On multivariable analysis (Table 2), receipt of AC was not associated with decreased hazards of death (HR $0.86,95 \%$ CI $0.64-1.15 ; p=0.31$ ) compared to observation alone among patients who were $\mathrm{pN}+$. When we added an interaction term of number of positive lymph nodes $\mathrm{x}$ treatment into this model, the term was not significant $(p=0.99)$, suggesting no effect modification of $\mathrm{AC}$ by number of positive $\mathrm{LN}$.

\section{DISCUSSION}

Non-organ confined disease at RC is associated with poor prognosis, with a 5-year OS of $25-38 \%[8,20,21]$. Patients who have patho- logic lymph node involvement despite being treated with NAC have a particularly poor prognosis with median overall survival estimates of 13-26 months [22-24]. Treatment options for these patients are limited to observation, use of additional chemotherapy or enrollment in clinical trials. Whether patients with persistent micrometastatic disease after systemic chemotherapy benefit from further systemic chemotherapy is unknown, yet many oncologists support administering additional chemotherapy [13].

Among 705 patients with pT3/4 or $\mathrm{pN}+$ disease at RC who were previously treated with NAC, $24 \%$ received subsequent $\mathrm{AC}$ while the rest were observed. We did not observe an improvement in survival for patients who received multi-agent $\mathrm{AC}$ in the entire cohort or on subgroup analysis of patients with $\mathrm{pN}+$. These observations suggest that additional multiagent AC for patients with positive lymph nodes after NAC may not be effective.

There have been two prior studies examining the effectiveness of AC for patients with lymph node metastases at RC following NAC. The M.D. Anderson Cancer Center reported on 37 patients who were treated with NAC or systemic chemotherapy from 1993-2003 for advanced or clinically lymph node positive bladder cancer and found to be $\mathrm{pN}+$ after RC [12]. Eleven patients (30\%) received AC, which was generally a platinum-based regimen that differed from what was given preoperatively. Patients selected to receive $\mathrm{AC}$ were younger and had a better performance status than patients who were observed. Median overall survival for the AC group was 16 months versus 12.6 months for the observation group, and $\mathrm{AC}$ was associated with improved recurrence free survival on multivariate analysis (HR $0.29,95 \%$ CI $0.10-0.81 ; p=0.02$ ), but no significant improvements in overall or disease-specific survival. However, 18 (49\%) patients from this cohort were treated with induction therapy for $\mathrm{cN}+$ disease rather than neoadjuvant chemotherapy.

The Moffitt Cancer Center recently reported on their experience with 88 patients with non-organ confined or LN positive disease after NAC and RC [7]. Adjuvant chemotherapy was administered in 29 of these patients while the rest were observed. The majority of the NAC and AC regimens were carboplatin or cisplatin based. The median cancer-specific survival was similar for the $\mathrm{AC}$ and observation cohorts (23 months versus 22 months, $p=0.65$ ), and $\mathrm{AC}$ was not associated with cancer-specific survival on multivariate analysis. On subgroup analysis of the 
Table 1

Demographic and clinical characteristics of the cohort

\begin{tabular}{|c|c|c|c|}
\hline Variable & $\begin{array}{c}\text { NAC only }(N=537) \\
N(\%)\end{array}$ & $\begin{array}{c}N A C+A C(N=168) \\
N(\%)\end{array}$ & $p$-value* \\
\hline \multicolumn{4}{|l|}{ Age } \\
\hline$<50$ & $36(7)$ & $21(12)$ & \multirow[t]{5}{*}{0.031} \\
\hline $50-59$ & $140(26)$ & $44(26)$ & \\
\hline $60-69$ & $197(37)$ & $68(40)$ & \\
\hline $70-79$ & $136(25)$ & $31(18)$ & \\
\hline$>80$ & $28(5)$ & $4(4)$ & \\
\hline \multicolumn{4}{|l|}{ Gender } \\
\hline Male & $357(66)$ & $113(67)$ & \multirow[t]{2}{*}{0.851} \\
\hline Female & $180(34)$ & $55(33)$ & \\
\hline \multicolumn{4}{|l|}{ Race } \\
\hline White & $480(89)$ & $154(92)$ & \multirow[t]{4}{*}{0.647} \\
\hline Black & $35(7)$ & $9(5)$ & \\
\hline Other & $18(3)$ & $5(3)$ & \\
\hline Unknown & $4(1)$ & $0(0)$ & \\
\hline \multicolumn{4}{|l|}{ Charlson/Deyo Score } \\
\hline 0 & $400(74)$ & $128(76)$ & \multirow[t]{3}{*}{0.903} \\
\hline 1 & $107(20)$ & $31(19)$ & \\
\hline$>1$ & $30(6)$ & $9(5)$ & \\
\hline \multicolumn{4}{|l|}{ Type of Facility } \\
\hline Academic/Research & $326(61)$ & $80(49)$ & \multirow{4}{*}{0.029} \\
\hline Comprehensive Community Cancer & $149(28)$ & $54(33)$ & \\
\hline Community Cancer & $24(5)$ & $13(8)$ & \\
\hline Other & $32(6)$ & $15(9)$ & \\
\hline \multicolumn{4}{|l|}{ Insurance Status } \\
\hline Private & $235(44)$ & $69(41)$ & \multirow[t]{6}{*}{0.078} \\
\hline Medicare & $247(46)$ & $69(41)$ & \\
\hline Medicaid & $26(5)$ & $17(10)$ & \\
\hline Uninsured & $22(4)$ & $8(5)$ & \\
\hline Other government & $4(1)$ & $4(2)$ & \\
\hline Unknown & $3(1)$ & $1(1)$ & \\
\hline \multicolumn{4}{|l|}{ Year of diagnosis } \\
\hline 2004-2008 & $141(26)$ & $46(27)$ & \multirow[t]{2}{*}{0.773} \\
\hline 2009-2013 & $396(74)$ & $122(73)$ & \\
\hline \multicolumn{4}{|l|}{ Tumor grade } \\
\hline Low & $18(4)$ & $1(1)$ & \multirow[t]{2}{*}{0.056} \\
\hline High & $445(96)$ & $142(99)$ & \\
\hline \multicolumn{4}{|l|}{$\mathrm{cN}$ stage } \\
\hline $\mathrm{cNO}$ & $453(84)$ & $133(79)$ & \multirow[t]{2}{*}{0.117} \\
\hline $\mathrm{cNX}$ & $84(16)$ & $35(21)$ & \\
\hline \multicolumn{4}{|l|}{ pT stage } \\
\hline$<\mathrm{pT} 1$ & $12(2)$ & $3(2)$ & 0.474 \\
\hline pT2 & $44(8)$ & $19(11)$ & \\
\hline$>$ pT3 & $473(90)$ & $146(87)$ & \\
\hline pN stage & & & \\
\hline pNO & $281(52)$ & $69(41)$ & 0.033 \\
\hline $\mathrm{pNX}$ & $14(3)$ & $4(2)$ & \\
\hline $\mathrm{pN}(+)$ & $242(45)$ & $95(57)$ & \\
\hline Histology & & & \\
\hline $\mathrm{UC}$ & $465(87)$ & $146(87)$ & 0.917 \\
\hline Non-UC & $72(13)$ & $22(13)$ & \\
\hline Number of LN examined & & & \\
\hline$<9$ & $135(28)$ & $41(28)$ & 0.972 \\
\hline$>10$ & $353(72)$ & $108(72)$ & \\
\hline Number of LN positive & & & \\
\hline$<2$ & $143(62)$ & $53(59)$ & 0.088 \\
\hline$>2$ & $89(38)$ & $37(41)$ & \\
\hline
\end{tabular}

${ }^{*} p$-values derived from chi-square tests for categorical variables and Students' $t$-test for continuous variables. 

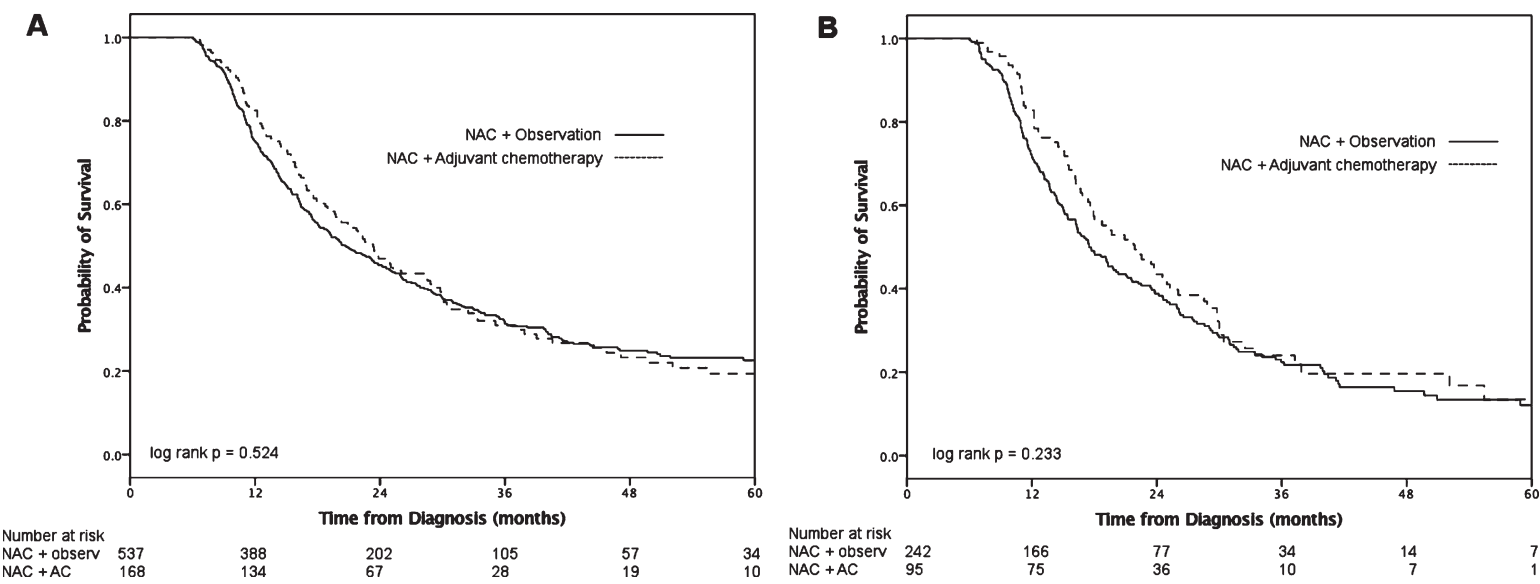

Fig. 2. Overall survival after NAC and RC for $\mathrm{pT} 3 / 4$ and/or $\mathrm{pN}+$ patients stratified by treatment group for all patients $(\mathrm{A})$ and $\mathrm{pN}+$ patients only (B).

Table 2

Multivariable analysis of risk of death after diagnosis with bladder cancer

\begin{tabular}{|c|c|c|c|c|}
\hline & $\begin{array}{c}\mathrm{pN}+\text { and/or pT3/4 }(\mathrm{N}=705) \\
\text { Adjusted HR }(95 \% \mathrm{CI})\end{array}$ & $p$-value & $\begin{array}{c}\mathrm{pN}+\text { alone }(\mathrm{N}=337) \\
\text { Adjusted HR }(95 \% \mathrm{CI})\end{array}$ & $p$-value \\
\hline Age (continuous) & $1.01(0.99-1.02)$ & 0.156 & $1.02(1.00-1.03)$ & 0.021 \\
\hline \multicolumn{5}{|l|}{ Sex } \\
\hline Male & 1.0 & ref & 1.0 & ref \\
\hline Female & $1.00(0.82-1.24)$ & 0.968 & $0.98(0.75-1.32)$ & 0.982 \\
\hline \multicolumn{5}{|l|}{ Race } \\
\hline White & 1.0 & ref & 1.0 & ref \\
\hline Non-white & $0.96(0.68-1.35)$ & 0.798 & $1.22(0.79-1.87)$ & 0.368 \\
\hline \multicolumn{5}{|l|}{ Charlson/Deyo score } \\
\hline 0 & 1.0 & ref & 1.0 & ref \\
\hline 1 & $1.23(0.97-1.58)$ & 0.093 & $1.31(0.94-1.82)$ & 0.108 \\
\hline$>1$ & $1.30(0.85-2.00)$ & 0.228 & $1.84(1.03-3.29)$ & 0.039 \\
\hline \multicolumn{5}{|l|}{ Pathologic $\mathrm{T}$ stage } \\
\hline$<\mathrm{T} 1$ & 1.0 & ref & 1.0 & ref \\
\hline $\mathrm{T} 2$ & $0.83(0.41-1.68)$ & 0.601 & $0.75(0.37-1.53)$ & 0.429 \\
\hline$>\mathrm{T} 3$ & $1.41(0.74-2.67)$ & 0.291 & $1.78(0.94-3.40)$ & 0.079 \\
\hline \multicolumn{5}{|l|}{ Histology } \\
\hline $\mathrm{UC}$ & 1.0 & ref & 1.0 & ref \\
\hline Non-UC & $1.30(0.99-1.70)$ & 0.058 & $1.34(0.9-2.02)$ & 0.152 \\
\hline \# LN examined (continuous) & $0.99(0.98-1.00)$ & 0.004 & $0.99(0.98-1.00)$ & 0.036 \\
\hline \multicolumn{5}{|l|}{ \# Positive LN } \\
\hline$<2$ & 1.0 & ref & 1.0 & ref \\
\hline$>2$ & $1.75(1.38-2.22)$ & 0 & $1.27(0.97-1.67)$ & 0.086 \\
\hline \multicolumn{5}{|l|}{ Treatment } \\
\hline NAC alone & 1.0 & ref & 1.0 & ref \\
\hline $\mathrm{NAC}+\mathrm{AC}$ & $0.91(0.72-1.14)$ & 0.422 & $0.86(0.64-1.15)$ & 0.31 \\
\hline
\end{tabular}

$47 \mathrm{pN}+$ patients, the AC group $(\mathrm{N}=24)$ had no survival advantage over the observation group.

We present the largest study examining $\mathrm{AC}$ in patients with advanced bladder cancer following RC and NAC. Similar to the study from Moffitt, we did not observe a benefit to $\mathrm{AC}$ when considering all patients with advanced disease. We hypothesized that patients with a lower burden of metastatic disease $(\leq 2$ lymph nodes) would benefit more from AC than those with a higher burden of disease. After stratifying by disease burden, neither patients with $\leq 2$ positive nor $>2$ positive lymph nodes who were treated with AC appeared to have a survival benefit and the interaction term in our multivariate analysis was not significant.

Residual disease after systemic chemotherapy suggests chemotherapy resistance and rechallenging patients with identical agents to their initial systemic therapy may not be effective $[25,26]$. A phase III trial comparing NAC and AC with MVAC compared to AC alone included 140 cT3-T4a patients with clinically 

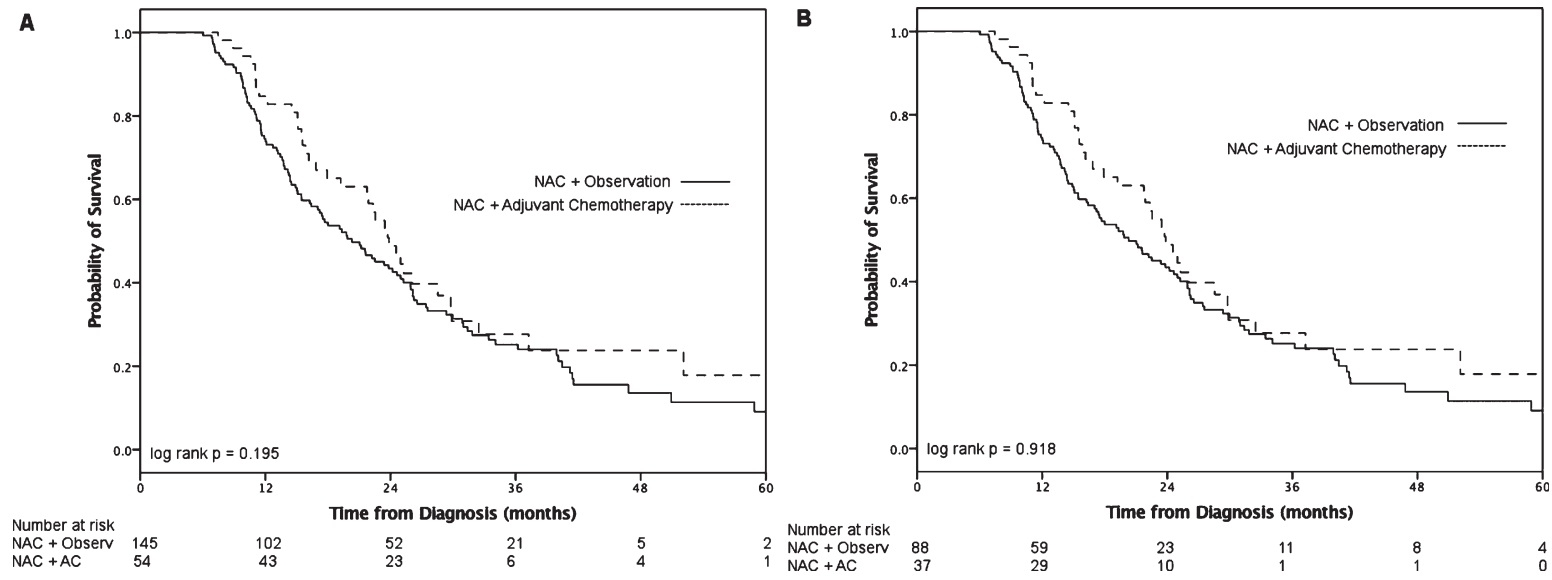

Fig. 3. Overall survival after NAC and RC with $(\mathrm{A}) \leq 2$ positive lymph nodes and (B) $>2$ positive lymph nodes.

negative lymph nodes, and found no difference in outcomes between the two groups [9]. Specifically, patients with advanced or $\mathrm{pN}+$ disease after two cycles of neoadjuvant cisplatin-based chemotherapy did poorly despite receiving additional postoperative chemotherapy.

A theoretical rationale for rechallenging patients with systemic chemotherapy may lie in sequential therapy, or administering a different agent than what was given preoperatively. Administering chemotherapeutic regimens that target disparate cell populations within a tumor may enhance the efficacy of a single multi-agent regimen and work to overcome chemotherapy resistance [27]. However, there is little data to suggest this approach is effective. In one phase II trial that included patients with high-risk or locally advanced bladder cancers, sequential therapy with two different multi-agent chemotherapy regimens downstaged $65 \%$ of patients to pT1N0 or less [25]. However, patients who did not respond well to the first line of chemotherapy and were switched to the second line early generally did poorly, suggesting that chemoresistant tumor biology may persist even in the face of a new agent. Another phase II trial of patients with locally advanced or metastatic urothelial carcinoma demonstrated promising results with sequential doxorobucin-gemcitabine (AG) and ifosphomidepaclitaxel-cisplatin (ITP), but it is unclear if the addition of AG significantly improved the results of ITP alone [26].

With NAC becoming increasingly utilized, patients with $\mathrm{pN}+$ disease after $\mathrm{RC}$ and NAC will be more frequently encountered [15]. Further research is required to determine which high-risk patients benefit from additional systemic therapy, and also which therapy is most effective. There have recently been promising results for use of immunotherapy for patients with metastatic disease [28], and trials of adjuvant immunotherapy for patients at high risk of recurrence after RC are currently underway [29, 30]. Furthermore, there is also a need to identify biomarkers that can identify patients who are less likely to respond to systemic cytotoxic chemotherapy. Such patients may be candidates for immediate surgery, immunotherapy or clinical trials.

Still, our findings are largely hypothesis generating given the absence of randomization and inability to account for unmeasured patient characteristics that are associated with treatment group selection. Reasons for utilizing AC are heavily influenced by patient factors that could not be accounted for, such as performance status, surgical complications, renal function and comorbidities [31]. Performance status is among the most important factors for deciding whether to use $\mathrm{AC}$, and this is not captured in the NCDB data [13]. Furthermore, AC may have been used in patients who were felt to have undetected lymph node involvement due to an insufficient lymphadenectomy.

Our study has additional important limitations. The type of chemotherapy was not available, though we limited our cohort to "multi-agent" chemotherapy, which are generally platinum-based regimens. While not considered standard perioperative chemotherapy, some patients may have been treated with carboplatin-based regimens, which are known to be inferior to cisplatin-based regimens [4, 32]. It was also unknown whether patients who received AC were rechallenged with different agents from the NAC setting. Another important limitation is the lack of information on the exact timing of postoperative 
chemotherapy after RC, the agents used, and the number of cycles given. Therefore, it is possible that some patients in the AC cohort received deferred, and not true "adjuvant," chemotherapy or instead developed metastatic disease and received salvage chemotherapy. Still, immediate AC and delayed chemotherapy may be associated with similar survival outcomes [11]. We excluded all patients with another primary cancer diagnosis as we could not identify the type of cancer and thus may have not have received chemotherapy for the indication of bladder cancer.

\section{CONCLUSIONS}

In our dataset of patients treated with NAC followed by RC, $24 \%$ of patients with residual nonorgan confined disease received additional AC. We did not observe a survival benefit among all patients with advanced disease after RC or in a subgroup of $\mathrm{pN}+$ patients. Clinical trials investigating adjuvant immunotherapy for these patients are currently underway.

\section{CONFLICT OF INTEREST}

None.

\section{REFERENCES}

[1] Mertens LS, Meijer RP, Meinhardt W, et al. Occult lymph node metastases in patients with carcinoma invading bladder muscle: Incidence after neoadjuvant chemotherapy and cystectomy vs after cystectomy alone. BJU Int 2014;114(1):67-74.

[2] Advanced Bladder Cancer Meta-analysis C. Neoadjuvant chemotherapy in invasive bladder cancer: A systematic review and meta-analysis. Lancet 2003;361(9373):1927-34.

[3] Grossman HB, Natale RB, Tangen CM, et al. Neoadjuvant chemotherapy plus cystectomy compared with cystectomy alone for locally advanced bladder cancer. N Engl J Med 2003;349(9):859-66.

[4] National Comprehensive Cancer Network: Clinical practice guidelines in oncology. https://www.nccn.org/ professionals/physician_gls/f_guidelines.asp-site

[5] Donat SM, Shabsigh A, Savage C, et al. Potential impact of postoperative early complications on the timing of adjuvant chemotherapy in patients undergoing radical cystectomy: A high-volume tertiary cancer center experience. Eur Urol 2009;55(1):177-85.

[6] Thompson RH, Boorjian SA, Kim SP, et al. Eligibility for neoadjuvant/adjuvant cisplatin-based chemotherapy among radical cystectomy patients. BJU Int 2014;113(5b):E17-21.

[7] Zargar-Shoshtari K, Kongnyuy M, Sharma P, et al. Clinical role of additional adjuvant chemotherapy in patients with locally advanced urothelial carcinoma following neoadjuvant chemotherapy and cystectomy. World J Urol 2016;34(11):1567-73.
[8] Manoharan M, Katkoori D, Kishore TA, et al. Outcome after radical cystectomy in patients with clinical $\mathrm{T} 2$ bladder cancer in whom neoadjuvant chemotherapy has failed. BJU Int 2009;104(11):1646-9.

[9] Millikan R, Dinney C, Swanson D, et al. Integrated therapy for locally advanced bladder cancer: Final report of a randomized trial of cystectomy plus adjuvant M-VAC versus cystectomy with both preoperative and postoperative M-VAC. J Clin Oncol 2001;19(20):4005-13.

[10] Leow JJ, Martin-Doyle W, Rajagopal PS, et al. Adjuvant chemotherapy for invasive bladder cancer: A 2013 updated systematic review and meta-analysis of randomized trials. Eur Urol 2014;66(1):42-54.

[11] Sternberg CN, Skoneczna I, Kerst JM, et al. Immediate versus deferred chemotherapy after radical cystectomy in patients with pT3-pT4 or N+ M0 urothelial carcinoma of the bladder (EORTC 30994): An intergroup, open-label, randomised phase 3 trial. Lancet Oncol 2015;16(1):76-86.

[12] Kassouf W, Agarwal PK, Grossman HB, et al. Outcome of patients with bladder cancer with $\mathrm{pN}+$ disease after preoperative chemotherapy and radical cystectomy. Urology 2009;73(1):147-52.

[13] Tan WS, Lamb BW, Payne H, et al. Management of nodepositive bladder cancer after neoadjuvant chemotherapy and radical cystectomy: A survey of current UK practice. Clin Genitourin Cancer 2015;13(3):e153-8.

[14] Milowsky MI, Rumble RB, Booth CM, et al. Guideline on muscle-invasive and metastatic bladder cancer (European association of urology guideline): American society of clinical oncology clinical practice guideline endorsement. J Clin Oncol 2016;34(16):1945-52.

[15] Reardon ZD, Patel SG, Zaid HB, et al. Trends in the use of perioperative chemotherapy for localized and locally advanced muscle-invasive bladder cancer: A sign of changing tides. Eur Urol 2015;67(1):165-70.

[16] Eble JN, Sauter G, Epstein JI. World Health Organization Classification of Tumors: Pathology and Genetics of Tumors of the Urinary System and Male Genital Organs. Lyon, France: ARC Press; 2004.

[17] American College of Surgeons. The National Cancer Database 2013 PUF Data Dictionary. http://ncdbpuf. facs.org/ (accessed 01/01/2016).

[18] Clifton MM, Psutka SP, Boorjian SA, et al. Cancer-specific mortality following radical cystectomy for bladder cancer with lymph node involvement: Impact of pathologic disease features and adjuvant chemotherapy. World J Urol 2015;33(3):373-9.

[19] Mills RD, Turner WH, Fleischmann A, et al. Pelvic lymph node metastases from bladder cancer: Outcome in 83 patients after radical cystectomy and pelvic lymphadenectomy. J Urol 2001;166(1):19-23.

[20] Stein JP, Cai J, Groshen S, et al. Risk factors for patients with pelvic lymph node metastases following radical cystectomy with en bloc pelvic lymphadenectomy: Concept of lymph node density. J Urol 2003;170(1):35-41.

[21] Abdel-Latif M, Abol-Enein H, El-Baz M, et al. Nodal involvement in bladder cancer cases treated with radical cystectomy: Incidence and prognosis. J Urol 2004; 172(1):85-9.

[22] Tully CM, Bochner BH, Dalbagni G, et al. Gemcitabinecisplatin (GC) plus radical cystectomy-pelvic lymph node dissection (RC-PLND) for patients (pts) with muscleinvasive bladder cancer (MIBC): Assessing impacts of neoadjuvant chemotherapy (NAC) and the PLND. J Clin Oncol 2014;32(suppl 4):abstr 355. 
[23] Sonpavde G, Goldman BH, Speights VO, et al. Quality of pathologic response and surgery correlate with survival for patients with completely resected bladder cancer after neoadjuvant chemotherapy. Cancer 2009;115(18):4104-9.

[24] Zargar H, Zargar-Shoshtari K, Lotan Y, et al. Final pathological stage after neoadjuvant chemotherapy and radical cystectomy for bladder cancer-does pT0 predict better survival than $\mathrm{pTa} / \mathrm{Tis} / \mathrm{T} 1$ ? J Urol 2016;195(4):886-93.

[25] Siefker-Radtke AO, Dinney CP, Shen Y, et al. A phase 2 clinical trial of sequential neoadjuvant chemotherapy with ifosfamide, doxorubicin, and gemcitabine followed by cisplatin, gemcitabine, and ifosfamide in locally advanced urothelial cancer: Final results. Cancer 2013;119(3):540-7.

[26] Milowsky MI, Nanus DM, Maluf FC, et al. Final results of sequential doxorubicin plus gemcitabine and ifosfamide, paclitaxel, and cisplatin chemotherapy in patients with metastatic or locally advanced transitional cell carcinoma of the urothelium. J Clin Oncol 2009;27(25):4062-7.

[27] Norton L. Conceptual and practical implications of breast tissue geometry: Toward a more effective, less toxic therapy. Oncologist 2005;10(6):370-81.
[28] Powles T, Eder JP, Fine GD, et al. MPDL3280A (anti-PDL1) treatment leads to clinical activity in metastatic bladder cancer. Nature 2014;515(7528):558-62.

[29] A Phase III Study of Atezolizumab Treatment Versus Observation as Adjuvant Therapy in Patients With PDL1 Positive, High Risk Muscle Invasive Bladder Cancer After Cystectomy [IMvigor010]. 2016. https://clinicaltrials. gov/ct2/show/NCT02450331 (accessed 6/1 2016).

[30] An Investigational Immuno-therapy Study of Nivolumab, Compared to Placebo, in Patients With Bladder or Upper Urinary Tract Cancer, Following Surgery to Remove the Cancer (CheckMate 274). 2017. https://clinicaltrials. gov/ct2/show/NCT02632409 (accessed 5/1 2017).

[31] Herr HW, Dotan Z, Donat SM, et al. Defining optimal therapy for muscle invasive bladder cancer. J Urol 2007;177(2):437-43.

[32] Galsky MD, Chen GJ, Oh WK, et al. Comparative effectiveness of cisplatin-based and carboplatin-based chemotherapy for treatment of advanced urothelial carcinoma. Ann Oncol 2012;23(2):406-10. 